\title{
Prévention des inondations en Europe : hydraulique, assurances, ou solidarité ?
}

\author{
Flood prevention in Europe : hydraulics, insurance or solidarity?
}

\author{
par Bernard Barraqué ${ }^{1}$, \\ DR CNRS, LATTS-ENPC
}

In developed countries, the Welfare State developed a flood control policy based on structural measures, which finally reached their own limits. This allowed in turn for a come back of liberal policies based on voluntary (and sometimes compulsory) insurance, which also have recently shown their limits. In Europe, in accordance with the general trend to consider water resources as a common property (i.e. with a status beyond the public vs private debate), what is sought is preventative action based on solidarity at the territorial level of the risk. What is at stake is then the construction of community and subsidiary sharing of information to allow risk appropriation by those who face it.

\section{I — INTRODUCTION}

Les phénomènes catastrophiques comme les inondations font l'objet d'une attention croissante dans le monde. Le développement des systèmes d'assurance conduit à mieux les répertorier, et par conséquent à réévaluer leurs conséquences économiques à la hausse. La compagnie de réassurance Munich a par exemple répertorié 22 grands désastres dans le monde entre 1990 et 1996 , avec plus de 1000 morts, ou plus d'un milliard de dollars de dégâts. On a atteint 140000 morts au Bangladesh en avril 1991, et respectivement 26,5 et 16 milliards de dollars en Chine l'été 1996 et aux Etats-Unis l'été 1993. Mais ce sont surtout les plus petits événements, avec très peu de pertes de vies humaines, et des dégâts matériels modérés, qui sont mieux connus, dans la mesure où leurs victimes arrivent à obtenir des compensations des assurances ou des gouvernements. On semble sortir du registre de la force majeure, pour rentrer insensiblement dans celui de la solidarité. Mais quelle solidarité : celle du Welfare State ou celle de l'assurance volontaire ?

Ce n'est pas un hasard si des compagnies d'assurances travaillent sur les catastrophes, car elles se sentent concernées par cette évolution, et se demandent ce qui se passera si une catastrophe majeure survient alors que les sociétés auront été habituées à une prise en charge. Dans son dernier rapport ${ }^{2}$, la Munich Re promet des surprises désagréables lors de la publication des comptes des réassureurs en mars
2000, et met leur préoccupation sur le compte de l'augmentation de la fréquence des événements assurés, qui mange peu à peu leurs réserves, alors qu'on entrevoit la possibilité d'une catastrophe dépassant les 50 milliards de $\$$ aux EtatsUnis. Dans ce pays, alors qu'on estime la dépense annuelle moyenne du niveau fédéral à 7 milliards de $\$$ en préparation et dédommagements, on sent que les politiques ont un effet pervers, en particulier parce que le niveau fédéral encourage indirectement par ailleurs un développement économique dans les zones à risque et sans suffisamment de précautions particulières au niveau des constructions (voir la synthèse critique [1]). En France, le gouvernement a discrètement augmenté de trois points le pourcentage de la surprime "cat' nat"' pendant l'été 1999.

Il n'y a pas d'évidence absolue que le nombre ou la sévérité des événements climatiques extrêmes aient crû, et ce qui semble plutôt en cause est l'augmentation de la vulnérabilité, en particulier aux inondations, du fait que le développement économique privilégie les côtes ou les rives des fleuves. Par ailleurs, la communauté scientifique n'est pas encore certaine d'un changement climatique à venir. Mais on pense généralement que s'il se produit, il se traduira par une

1 Colloque Inondations, SHF Sept. 1999. Je remercie ici les collègues avec qui cette réflexion s'est développée : Claude Gilbert du programme risques et situations de crise du CNRS ; Bernard Chastan et Pierrick Givone du CEMAGREF Lyon, et d'autres collègues du réseau Euraqua : et Patricia Gressent, qui fait sa thèse sur ce sujet.

2 Voir les Echos du 4 Novembre, et Le Monde du 9 Novembre 1999. 
violence accrue des phénomènes, ce qui ne fait que renforcer la problématique des coûts croissants pour la société, et un sens élémentaire de prudence appelle à compléter les mesures hydrauliques classiques par d'autres approches, visant les populations soumises au risque. On sait d'ailleurs aujourd'hui que les mesures structurelles, comme les barrages-réservoirs en amont, ne sont pas efficaces précisément pour les plus grands événements. Et en ce qui concerne l'Union européenne, la DG XVI de la Commission, chargée des fonds structurels et régionaux, a lancé une réflexion sur la maîtrise des phénomènes climatiques extrêmes (inondations et sécheresses) dans la partie méditerranéenne de l'Europe, dans l'espoir que le développement d'actions d'aménagement du territoire préventives réduirait le coût des mesures structurelles. Mais, dans le même temps justement, la construction européenne et le principe libéral d'ouverture à la concurrence qui y prévaut conduit à remettre en cause les divers systèmes d'assurance ou de solidarité fiscale imposées existant ici et là. La recherche de nouvelles politiques publiques se fait dans un contexte d'incertitude.

On défend ici l'idée que ce n'est pas le changement du risque ni sa meilleure connaissance qui conduit à ces discussions, mais plutôt le changement de style des politiques publiques en cette fin de siècle. Aux yeux des sociologues et des analystes des politiques publiques, les politiques territoriales et d'environnement semblent presque avoir été inventées pour poser la question de la gouvernance, c'est-à-dire de la décision publique quand le pouvoir de décision est morcelé entre de nombreux acteurs. C'est typique lorsque l'on veut passer de l'élimination du risque (comme dans les politiques sanitaires du siècle dernier), à sa gestion. Mais la montée de l'environnement correspond aussi à celle de la gestion communautaire et solidaire de common properties (à traduire par patrimoines communs), notamment parce qu'aux Etats-Unis où la politique correspondante a débuté, le communautarisme a toujours constitué le principal frein au libéralisme triomphant. C'est ainsi que le courant d'économie des ressources naturelles (fondé par l'Américain d'origine allemande Ciriacy Wantrup [2]), s'est constitué dès avant le fameux article de Hardin [3] sur la tragédie des communaux, qui ne voit de solution à leur surexploitation que dans leur morcellement et leur appropriation privative. La notion de patrimoine commun correspond en fait à des biens se situant mal dans la confrontation public-privé, dominante en système libéral, qui est fondée sur l'appropriation privative au sens juridique, ou inversement sur la domanialisation publique. Dans le cas des inondations, cela signifie qu'on doit chercher d'autres solidarités que celles de l'assurance volontaire de la propriété privée, et de l'assurance collective de l'hydraulique structurelle. On essaie de remplacer le principe d'appropriation par celui de l'usage non appropriatif des sols et des ressources naturelles, et de faire une planification participative en partant de la culture des usagers du bien en patrimoine, et en la développant, plutôt que de conduire une démarche traditionnelle de planification descendante. Et cela d'autant plus qu'on a eu le temps de se rendre compte, notamment dans les pays latins de droit romain, que les servitudes d'urbanisme imposées dans les plans traditionnels élaborés selon des règles nationales sont assez mal perçues et respectées par les responsables et les résidents des collectivités locales [4,5]. En Europe comme en particulier en France, l'eau joue un rôle essentiel dans le développement de cette conception patrimoniale.

\section{II — L'EAU, PATRIMOINE COMMUN EN EUROPE}

Une analyse comparative des politiques et de l'administration de l'eau dans cinq pays membres, financée par la DG $\mathrm{XII}^{3}$, a permis de reconstituer l'histoire contemporaine du droit de l'eau, et de faire l'hypothèse suivante : alors qu'au niveau mondial, les ressources en eau et les services publics d'eau potable et d'assainissement font l'objet d'un débat relatif à la privatisation ou à la gestion publique, en Europe, la confrontation public-privé serait de plus en plus brouillée dans les deux secteurs de la politique (ressource, services). D'une part, on voit bien se développer une infinité de nuances dans le partenariat public privé pour la gestion des services : cela va de la privatisation totale à l'anglaise, avec régulateur fort au niveau central, et donc centralisation, à la privatisation formelle de la gestion publique en fait maintenue comme aux Pays Bas et en Allemagne.

C'est dans le domaine de la ressource que les évolutions seraient les plus frappantes : l'eau serait considérée de plus en plus comme un patrimoine commun (common property en anglais), avec une réduction de la part de l'appropriation privée de l'eau, et l'émergence d'une nouvelle structuration en tension, entre domanialisation, réglementation centralisée et planification d'un côté, et gestion communautaire et contractuelle, territorialisée plus localement, de l'autre.

Paradoxalement, cette nouvelle structuration ramènerait de fait la gestion de l'eau à ses racines anciennes du haut Moyen Age : en effet à cette époque, le statut de l'eau était celui du partage coutumier entre les usagers, bien sûr dans le droit coutumier des tribus germaniques (Markgenossenschaft), mais aussi dans le droit Romain, du moins pour une partie de la ressource, à savoir les eaux courantes non publiques. Renvoyons ici rapidement à la synthèse impressionnante de Dante Caponera [6] sur l'histoire du droit de l'eau dans le monde entier. Il distingue bien sûr les deux sources du droit européen, coutumes germaniques, et droit romain écrit. Mais il caractérise ce dernier ainsi :

"En simplifiant, les ressources en eau étaient réparties en trois catégories dans le droit romain :

(i) les eaux communes à tous (res comunis omnium), ne pouvant faire l'objet d'aucune appropriation. Personne ne pouvait posséder ces eaux, qu'il soit un individu, une communauté ou l'État ; avec l'air et le rivage maritime, elles ne pouvaient faire l'objet que de droits d'usage. Toutes les eaux courantes étaient dans cette catégorie".

"(ii) les eaux publiques (res publica), appartenant à une collectivité, une municipalité, ou une autre institution. L'usage de ces eaux était réservé aux institutions, qui avaient sur elles un droit fondé en titre, et qui pouvaient à leur tour accorder un droit d'usage à d'autres".

"(iii) les eaux privées, appropriées privativement ${ }^{4}$, qui ne constituaient qu'une petite partie des eaux : eau de pluie, eaux souterraines, et tout petits cours d'eau. La propriété de l'eau était généralement attachée à celle de la terre. Le pro-

\footnotetext{
3 II s'agit de la recherche Eurowater, réunissant des équipes du Portugal, du Royaume Uni des Pays Bas, de France et d'Allemagne. Le financement de la D.G. XII a été complété par des institutions des pays concernés, comme par exemple le LAWA en Allemagne, les Agences de l'eau en France, la fondation Gulbenkian au Portugal ... Le travail spécifique repris ici est le chapitre 11 du volume II d'Eurowater, publié par Balkema en 1998 [19]
}

4 En fait, il s'agit souvent de biens considérés comme res nullius, dont on laissait l'appropriation aux proprićtaires des terrains. 
priétaire avait un droit d'user (et d'abuser) exclusif et illimité sur ces eaux, sans restriction, indépendamment des conséquences de cet usage sur les fonds voisins (ius utendi et abutendi). Dans le droit romain, ce droit de propriété et d'usage sur l'eau était nécessairement limité par les droits similaires des propriétaires voisins, ainsi que par les droits accordés à des tiers par l'État. L'eau était donc considérée comme partie intégrante de la terre, et le propriétaire pouvait disposer librement de l'eau qu'il trouvait sur son fonds, sans avoir de considération pour ses voisins ; inversement, il pouvait subir le même traitement de leur part".

En fait, on peut reconnaître là les trois catégories du droit de l'eau dans notre pays, telles que reprises dans la loi de 1898 sur le régime et la répartition des eaux. Mais en quoi peut-on dire que la res comunis omnium devient maintenant une catégorie dominant les deux autres ? Revenons en arrière : avec le système féodal, un système très complexe s'est développé, puisque les seigneurs avaient droit de disposer et d'abuser de l'eau (domaine éminent), et qu'ils pouvaient donner leur droit en tenure à leurs vassaux ; alors que les communautés paysannes n'avaient que droit d'user sans abuser (domaine utile), mais avec des droits souvent plus anciens que ceux des seigneurs, et que ceux-ci devaient respecter. A l'époque classique, l'avènement des grandes monarchies et des Etats nations a correspondu à la ré-émergence de l'eau publique des Romains, et des eaux privées. II en résultait un enchevêtrement de droits, dont le Code civil de Napoléon, et ses succédanés dans de nombreux autres pays, ont tenté de sortir par la voie libérale, c'est-à-dire en tentant de n'avoir plus que des eaux publiques et des eaux privées, comme le reste des ressources d'ailleurs. Ce fut l'époque de la réduction des "communaux". Mais en ce qui concerne l'eau, la simplification libérale n'a jamais abouti, notamment parce que dans certaines situations, il n'y avait pas de meilleure alternative que la gestion communautaire (zones de marais, et inversement zones arides où l'irrigation est essentielle).

Puis est venue l'époque de la modernisation de l'Etat, l'avènement du welfare State, le New deal, les expériences planificatrices des dictatures fascistes et staliniennes : à partir des années 1930, un peu partout dans le monde développé, l'accent est mis à la fois sur une domanialisation accrue de l'eau, et sur ce qu'elle permet, la grande hydraulique, et même les transferts inter-bassins, qui requièrent la souveraineté étatique. Dans le monde développé, y compris aux Etats-Unis, cette période va des années 1930 à l'émergence du thème de l'environnement. En France par exemple, c'est en 1935 que le Président du Conseil Pierre Laval prend les décrets permettant d'une part de domanialiser les eaux souterraines en dessous de $30 \mathrm{~m}$ en cas de sur-exploitation, et d'autre part de limiter les constructions dans les lits majeurs des rivières navigables pour laisser passer les inondations (PSS). Et à plusieurs reprises, on discute de la domanialisation complète de l'eau, notamment lors des débats de la loi sur l'eau de 1964 au Sénat avec Pierre Marcilhacy. On recule, en fait, à cause du coût de l'expropriation des droits acquis. Et puis les choses s'inversent en partie, car si le pouvoir de réglementation du gouvernement central continue à se développer, et jusque dans la loi de 1992, en fait la politique de l'environnement conduit à remettre en cause la sectorisation centralisante de la gestion de l'eau, au profit d'une re-territorialisation dans la gouvernance. Le fleuron de cette politique est bien sûr la création et le développement des agences de l'eau, dont la mission historique est peut-être d'obtenir par la voie économique ce qui est impossible par la voie juridique : les servitudes d'aménagement ne font pas l'objet d'une compensation (selon la logique de l'Etat planificateur au-dessus des citoyens), mais la propriété privée est si bien défendue qu'elle bloque la planification. Le système des redevances et des aides réintroduit des compensations réciproques entre des acteurs qui sont redéfinis en termes d'usage de l'eau et non d'appropriation. Aujourd'hui, la réaffirmation de l'importance de la participation des usagers correspond à une recherche d'efficacité qui passe en partie par la remise en cause de la souveraineté de la propriété privée, et même de la propriété de l'Etat. Beaucoup d'autres pays membres ont donné une base juridique aux communautés d'usagers de l'eau, ou en ont fait des institutions.

Dans un article faisant le point sur les grandes évolutions récentes du droit de l'eau, Stefano Burchi [7], de la FAO, constate une montée générale des approches de planification régionale de l'eau, ainsi que des procédures d'autorisation préalable pour les usages industriels. Il en résulte "un rôle marginalisé des eaux appropriées privativement et des droits acquis", y compris pour les eaux souterraines dans les pays latins en Europe, et même aux États-Unis. L'auteur voit également, et comme nous, une tendance à la centralisation dans le mouvement même de privatisation de l'eau du RoyaumeUni. Mais il constate également que se développent des systèmes de redevance et d'incitation économique, qui facilitent les compromis entre l'intérêt général et les intérêts particuliers : "afin d'éviter de longues et incertaines batailles au tribunal, et pour développer en même temps l'effectivité des réglementations restreignant les droits d'eau, la législation récente s'est tournée vers les incitations économiques et fiscales - qui forment une sorte de compensation par avance - conduisant les usagers du sol à accepter des obligations auxquelles ils auraient résisté sans cela". L'auteur est donc sur une ligne voisine de la nôtre, bien qu'il ne dise rien sur la question du patrimoine commun. Plus généralement, il récuse l'interprétation selon laquelle les eaux publiques sont nécessairement appropriées par l'Etat : "les eaux courantes sont publiques à cause de leur aptitude à faire l'objet d'usages publics, mais cela n'entraîne pas forcément leur appropriation publique. La propriété d'Etat est en ce sens clairement une fausse conception, car ce qui compte est la gouvernance de la ressource par un Etat-gardien, pas son appropriation par un Etat-maître. Ce qui compte, en théorie juridique et en jurisprudence, c'est le droit d'usage de la ressource, et c'est à travers la fonction d'accorder ce droit que l'Etat gardien accomplit sa tâche primordiale, celle de protéger la ressource dans l'intérêt des citoyens ...".

Cette longue discussion de l'évolution contemporaine du droit de l'eau n'était pas gratuite. Elle a été faite ici parce que le domaine des inondations n'y échappe pas.

\section{III — LES LIMITES DES POLITIQUES STRUCTURELLES}

Comme on l'a dit, les Etats sont de plus en plus intervenus dans l'économie nationale après la grande crise, et ils l'ont fait par la réalisation d'infrastructures. Notamment, les barrages-réservoirs, imaginés dès le XIXe siècle comme moyens de lutter contre les inondations, ont enfin été réalisés, parce qu'ils permettaient de sécuriser des ressources de plus en plus convoitées par divers types d'usagers indus- 
triels, et que leur rentabilité était de ce fait mieux assurée, par un besoin positif d'eau. Mais, pour emporter des décisions coûteuses, on sait que rien ne vaut l'urgence d'une bonne catastrophe, et les inondations catastrophiques ont souvent servi d'argument final pour la décision. Mais a contrario, cela a entraîné des effets pervers. En effet, les barrages paraissaient plus fiables que les anciens systèmes locaux des digues toujours renforcées, tout en changeant l'échelle territoriale de la politique publique. Et les faibles taux d'intérêts de l'après-Guerre facilitaient les grands investissements nationaux. Mais la conséquence indirecte a été de rendre les gouvernements "responsables" aux yeux du public en cas d'inondations catastrophiques. En effet, les mesures structurelles et la modélisation planificatrice ont changé la problématique des événements majeurs, en les faisant sortir du registre de la fatalité et de la "force majeure", pour les faire rentrer dans celle du welfare et de la solidarité nationale, où les citoyens ont l'illusion que l'Etat maîtrise les risques. Cette dé-responsabilisation locale se retourne contre l'Etat lorsqu'un événement majeur dépasse les capacités hydrauliques, puisque l'Etat est alors tenu pour responsable ${ }^{5}$.

Bien sûr, la modélisation a permis de mieux connaître les zones à risque, et d'imposer des contraintes à l'urbanisation, en contrepartie ou en complément des mesures structurelles. Mais il faut bien admettre que ces mesures foncières ont souvent échoué devant la résistance des collectivités locales et des citoyens de base. Car il est bien connu qu'en cas de risque faible, on tend à sous-estimer l'espérance mathématique de ses pertes. Les collectivités locales, qui ont en charge l'aménagement, favorisent le développement économique au détriment de l'environnement et des risques, et cherchent ensuite à obtenir des gouvernements des investissements supplémentaires de protection, en profitant des rapports de force entre centre et périphérie, bien analysés par la Science politique française [8]. Mais de plus en plus la résistance des populations des régions concernées par les barrages amont a fait monter le coût politique, et bien sûr économique, des réservoirs, ce qui en a limité l'intérêt.

Une fois que la population a commencé à s'installer dans la zone à risque, la situation devient encore plus compliquée, parce que si les élus locaux établissent des contraintes à la construction, ceux qui sont déjà installés voient leur bien perdre de sa valeur, au point parfois qu'ils ne peuvent plus déménager. Pour les protéger, les responsables ne rendront pas les risques publics, et il sera alors difficile d'empêcher de nouveaux venus de s'installer. Cela crée un phénomène de cercle vicieux, surtout dans les pays où des systèmes de surprime d'assurance obligatoire permettent aux élus locaux d'obtenir plus facilement le classement de l'événement climatique dans ceux soumis à indemnisation étatique. C'est ainsi que le grand projet des années 1950-60 d'éliminer le risque par l'hydraulique a relativement conduit à une impasse. Seuls les Hollandais semblent arriver à faire respecter leurs plans d'urbanisme, mais ils sont presque tous dans l'une des plus grandes zones inondables d'Europe !

Malheureusement, les dispositifs de planification restent en décalage par rapport à cette prise de conscience. Burby et al. [1] mettent en cause le développement économique local dans les zones à risque, mais en dernière analyse, ils pensent que c'est le niveau fédéral qui est le principal responsable, car il a indirectement encouragé cette politique. De plus aujourd'hui, il constitue plutôt un obstacle qu'une aide à l'inversion de tendance, qui permettrait de réduire les risques par la prévention locale dans les plans de risque. Notamment parce qu'en prenant en charge les dégâts, il incite en fait les acteurs locaux à prendre des risques. En France on constate la même chose du fait du décalage spatial entre l'échelle de la solidarité (nationale) et celle des plans de risque (locale). Et si l'on se tourne vers des pays émergents comme le Brésil, on se trouve devant une cacophonie extraordinaire, parce que les jeux d'acteurs sont doublement amplifiés [9] : d'une part l'aide internationale se concentre sur de grands projets aussi visibles que peu efficaces et surtout trompeurs en termes d'élimination du risque ; et d'autre part, la modernisation de l'agriculture laisse une population croissante de miséreux sans terre qui vont s'installer là où ils peuvent, c'est-à-dire dans les zones inondables.

\section{IV — LES LIMITES DES POLITIQUES D'ASSURANCE VOLONTAIRE}

Les années 1980 ont amené un recul général de l'action des gouvernements. La crise économique rend l'argent trop cher pour être gaspillé dans des plans de solidarité sans contrepartie, et la grande hydraulique est évidemment concernée. Y compris chez les hauts fonctionnaires, on a vu se développer le discours alternatif de la responsabilité individuelle des preneurs de risque : puisqu'ils prennent des risques, qu'ils paient leur assurance en conséquence ! Un discours de ce type a été tenu par le Premier ministre polonais au moment où la gigantesque et exceptionnelle crue de l'Oder a commencé [10]. Si dans ce cas extrême la phrase était particulièrement maladroite, elle montre bien comment un discours libéral peut très rapidement remplacer un discours étatiste, tout en restant enfermée dans le cadre mental de la confrontation public-privé.

Les politiques de maîtrise des inondations reposant sur l'assurance rencontrent plusieurs difficultés. D'abord, elles constituent une régression de l'approche préventive vers une approche curative, dans la mesure où les compagnies d'assurances ne peuvent forcer directement leurs clients à faire de la prévention. Et, on l'a déjà souligné, les assurés sous-estiment les conséquences d'événements extrêmes avec probabilité très faible. Mais le pire est que dans un système volontaire, la plupart des gens ne sont pas vraiment assurés contre les catastrophes, et cela pour la même raison.

La raison en est d'abord que les compagnies d'assurance rechignent à couvrir des risques qu'elles ne peuvent pas quantifier. C'est la raison profonde de la notion de force majeure. Lorsqu'elles prennent le risque d'assurer les catastrophes, elles augmentent les primes considérablement, ce qui conduit une partie de leur clientèle à renoncer, soit pour des raisons économiques, soit par un phénomène de défiance accrue. S'il y a moins d'assurés, alors le risque s'accroît pour la compagnie d'assurances, qui doit à nouveau augmenter ses primes. Cela constitue un autre cercle vicieux, et d'ailleurs la même enquête de la Munich Reinsurance [11] montre qu'au moment des grandes inondations, une faible part des biens est généralement assurée. Dans certains pays la difficulté est contournée par des assurances obligatoires ou par des fonds du gouvernement. En Allemagne, par

5 Citons Peter Borrows, Regional Flood Defence Officer de l'Agence de l'Environnemen anglaise, région Tamise : " l'année dernière, nous avons eu 5 morts au printemps, et 2 autres en octobre. Maintenant l'Agence est le point focal de toutes les critiques, que ce soit justifié ou non. Nous allons être rendus responsables de chaque nouvelle inondation." 
exemple, la crue du Rhin de 1993-94 a causé des dommages évalués à 1,3 milliard de DM, alors que seule une valeur de 300 millions de DM était assurée (Potmann \& Gerhart, in [11]). Lors de l'inondation similaire de l'année suivante, les dommages n'ont été que de 500 millions DM, ce qui indique qu'il y a eu un processus d'apprentissage. Pour la crue de 1993, les dommages semblent avoir été mieux couverts dans le Land de Bade-Wurtemberg, où les propriétaires fonciers sont obligés de cotiser à une assurance monopolistique d'Etat comprenant l'inondation : rien que sur les bâtiments, le remboursement a atteint 180 millions DM. Or le monopole de l'assurance a été interdit par une Directive européenne le $1^{\text {er }}$ juillet 1994 , ce qui a entraîné un intense débat en Allemagne : aux défenseurs de l'acceptabilité sociale de l'assurance, qui constatent que le monopole d'Etat fait baisser des primes des assurés, répondent ceux qui pensent qu'il n'est pas juste que la grande majorité des citoyens, qui ne prend pas de risques, doive payer pour le petit nombre qui en prend. En Finlande, la politique de compensation des dommages est très différente : il n'y a pas d'assurance catastrophe naturelle, mais le gouvernement gère un budget de compensation des dommages fixé à l'avance. L'argent disponible est alors réparti chaque année entre tous ceux qui ont subi plus qu'un certain seuil de dommages cette année là, lors d'événements dépassant la période de retour de 20 ans. Selon le nombre de catastrophes dans l'année, le taux de couverture varie de 40 à $80 \%$ (Vehviläinen et al., in [11]).

Une comparaison européenne détaillée des systèmes en vigueur, de leur mise en œuvre et de leur efficacité, devrait constituer une priorité. Et il y a bien des enseignements à tirer des Etats Unis : la crue du Mississipi de 1993 offre un exemple particulièrement intéressant. En effet, les Américains ont tenté de résoudre le problème d'une couverture insuffisante des risques majeurs en créant la FEMA (Federal Emergency Management Agency), qui notamment gère un fonds d'assurance spécial subventionné par le gouvernement. Les gens vivant dans des zones à risque peuvent souscrire cette assurance spéciale, à condition que leur collectivité locale mette en place des mesures de réduction de la vulnérabilité, comme des zonages restreignant les droits de construire pour conserver et regagner des zones d'expansions de crue. Cependant, comme le fonds était supposé équilibrer ses aides avec les cotisations des souscripteurs, il semble avoir buté sur la question de la sous-estimation du risque. Ce qui a ensuite entraîné le cercle vicieux de l'augmentation des primes et du renoncement à l'assurance. En définitive, lorsque la grande crue catastrophique est arrivée, il semble que seulement $30 \%$ des personnes concernées étaient assurées.

En France, on a tenté de résoudre ce problème en élargissant la solidarité à l'extrême, c'est-à-dire en faisant payer une surprime à tous les assurés, qu'ils soient en zone de risque ou non. Les indemnisations en cas de catastrophe naturelle interviennent sur arrêté du Préfet suite à la demande des maires des communes concernées. Jusqu'à présent, les fonds collectés ont dépassé les indemnisations, car on a ajusté l'offre et la demande, et doublé le montant de la surprime au bout de quelques années (de 4,5 à $9 \%$ ). L'ensemble du système est sécurisé par l'implication de l'Etat en cas de dépassement des fonds disponibles (ce qui arriverait sûrement en cas de tremblement de terre à Nice). La plupart des arrêtés de cat'nat' sont cependant pris pour des inondations. Mais la récente augmentation de la surprime à $12 \%$, même si elle est justifiée par les conséquences indirectes des sécheresses sur la stabilité des bâtiments, n'indique-t-elle pas qu'on est en train de dériver, et que donc le système ne serait tenable qu'à court terme ?

Une évaluation faite par nos collègues de la Société Française pour le Droit de l'Environnement [12] montre d'abord que pour les victimes de catastrophes, le nouveau système constitue une amélioration considérable, puisqu'ils sont indemnisés au lieu d'être souvent simplement ruinés. De plus, comme on l'a mentionné, le système se montre économiquement viable, puisqu'il permet même bon an mal an de mettre de l'argent de côté pour un événement très grave. Cependant, nos collègues constatent aussi un risque de dérive : d'abord, ce sont de plus en plus des événements presqu'ordinaires, facilement évaluables, et pas trop chers, qui sont couverts. Ensuite, et surtout, le second volet de la loi de 1982 (les plans d'exposition aux risques) est beaucoup moins bien mis en æuvre. Cela tient à plusieurs raisons, dont la difficulté à évaluer précisément les risques. Mais ce qu'on peut légitimement craindre, c'est que les maires soient d'autant plus tentés de donner des permis en zone à risque qu'en cas d'inondation, les victimes seraient prises en charge par l'assurance à sa demande. On se retrouve là en fait dans un vieux schéma des politiques en France, analysé en termes de régulation croisée par divers auteurs [8] : la dérogation à la règle nationale fabrique un partage du pouvoir entre les élus locaux et les préfets, dans un jeu non transparent entre centre et périphérie.

Le gouvernement a admis ce risque, et à deux reprises, il a souhaité accélérer la procédure d'élaboration des P.E.R. : loi de 1988 qui rend obligatoire la publication des cartes de risque, et loi Barnier de 1995 redonnant plus d'initiative aux Préfets, et mettant les agences de l'eau à contribution pour financer les équipes réalisant les plans. Ce dernier point est d'ailleurs controversé, car les agences n'ont pas de base pour prélever des redevances correspondant au risque d'inondation, et surtout à son aggravation par des activités économiques (imperméabilisation des sols, suppression de zones d'expansion de crues, installation en zone à risque, etc.). Et, au moment où les factures d'eau ont augmenté au point d'être sur le devant de la scène, il est encore plus difficile de faire payer la politique de lutte contre les inondations dans les factures d'eau et d'assainissement, alors que légalement il est déjà interdit de facturer la gestion de l'assainissement pluvial. On risque fort de rester dans une situation évoluant trop lentement, et facilitant l'aggravation des risques à long terme.

Certains ont alors proposé de revenir à un système d'assurances plus classique, en revenant sur la loi de 1982 qui interdisait aux compagnies d'assurance d'augmenter les primes de ceux ayant subi des catastrophes naturelles, en bénéficiant de la connaissance progressivement accumulée. Et en effet, le système français est comme celui du Bade Wurtemberg, en contradiction avec la logique libérale de l'assurance comme contrat basé sur la volonté individuelle. La commission européenne peut à tout moment condamner notre dispositif comme contrevenant au principe de l'ouverture à la concurrence.

D'un autre côté, si l'on revient au contrat d'assurance classique, les compagnies d'assurance feront leur travail et augmenteront les primes des assurés à risque. On risque de 
se retrouver à moyen terme avec le même cercle vicieux qu'aux Etats-Unis, les plus pauvres abandonnant leur assurance, tout en se retrouvant bloqués dans des zones à risques. D'après Peter Borrows (voir note 5), les inondations de Pâques 1998 à Northampton ont frappé surtout des gens modestes qui n'étaient pas assurés, et qui n'ont donc pas reçu d'aide de l'Etat, celle-ci venant en complément du remboursement de l'assurance. D'où l'idée de créer une assurance spéciale, à primes réduites et à couverture faible, mais qui ouvrirait le droit à des aides de l'Etat.

\section{V — POUR UNE APPROCHE EN TERMES DE PATRIMOINE COMMUN}

La principale leçon du cas français est en fait qu'il est dangereux à terme de déconnecter le mécanisme de "solidarité forcée" organisé au niveau national, et la planification des zones de risque, organisée localement, car le curatif et le préventif sont alors séparés territorialement, ce qui ne facilite pas l'apprentissage et la prise de conscience. C'est ici qu'on peut reprendre le thème du patrimoine commun : si l'approche étatique basée sur l'offre hydraulique échoue, et que l'approche libérale basée sur la demande d'assurance échoue également, pourquoi faut-il organiser l'action sur une base communautaire ?

Dans la première partie de ce texte, on a rappelé l'importance croissante d'une définition de l'eau comme patrimoine commun, qui conduit à une régulation en termes d'usage plutôt que d'appropriation. Et Ciriacy Wantrup n'a pas manqué de répondre, à l'argument libéral de Garrett Hardin sur la "tragédie des communaux", que l'Europe offrait encore actuellement l'exemple de nombreux communaux fonctionnant parfaitement bien, et sans contradiction avec le système libéral dominant, parce que les règles de partage du patrimoine commun entre usagers étaient à la fois démocratiques et efficaces (voir aussi [17]). Mais cet argument de type anthropo-juridique peut aussi être présenté en termes économiques : lorsque de nombreux acteurs se partagent le pouvoir de décision, et sont dépendants des informations détenues par les autres, ainsi que de l'incertitude de situations non reproductibles où la décision ne peut pas être routinisée, les économistes disent que le "coût de transaction" est élevé. Il peut même dépasser la valeur économique de la décision. Dans ces conditions, on n'a guère d'autre possibilité que de tenter de constituer les acteurs en communauté solidaire, afin qu'ils échangent leurs informations volontairement, et qu'ils apprennent collectivement, ce qui réduit considérablement les coûts de transactions. Dans le cas des inondations, on sait que la modélisation hydrologique de bassins est très complexe, mais aussi qu'elle devient encore plus complexe lorsque le développement économique transforme en permanence les débits d'eau naturels. Il devient très difficile d'affecter les responsabilités des différents usagers de l'eau, ce qui pose problème aux économistes comme aux juristes. En général, même une excellente modélisation hydrologique risque de ne rencontrer que l'incompréhension des usagers, dans la mesure où ils se sentent mis devant le fait accompli par la modélisation. Dans une perspective d'acceptabilité socio-politique, la participation des usagers doit être organisée dès avant le travail de constitution des connaissances hydrologiques. C'est notamment l'un des enjeux majeurs de la gestion intégrée des rivières proposée par la Commission européenne dans la Directive cadre en projet : faut-il seulement prévoir une participation des usagers en fin de processus ?

C'est à partir de ces réflexions qu'on peut développer une analyse de ce qui rend nos agences de l'eau incontournables, au moment même où elles sont sous le feu des critiques [13, 18]. Elles sont typiquement des institutions subsidiaires et communautaires où les divers types d'usagers viennent apprendre ensemble (les uns des autres) la complexité de la politique à conduire, et ensuite négocier un partage solidaire des coûts de la reconquête du milieu. Seulement, cet apprentissage collectif est généralement un processus long, se déroulant à l'échelle de décennies. Les agences d'aujourd'hui sont d'ailleurs bien différentes de celles mises en place en 1970 : initialement elles devaient cantonner leur action à des "travaux d'intérêt commun", c'est-à-dire ni collectifs ni privés ; autrement dit elles ne pouvaient pas intervenir dans la construction des réseaux d'égout (intérêt collectif) ni de l'assainissement autonome (intérêt privé), mais seulement dans les stations d'épuration, ainsi que les barrages-réservoirs multi-fonctions (intérêt commun, c'est-à-dire responsabilité partagée et diffuse entre de multiples acteurs) [16]. C'est parce qu'au cours des années 1970, l'Etat a progressivement réduit son régime de subventions, que les agences de l'eau ont pris le relais : il est en effet inutile d'avoir une belle station d'épuration, si le réseau d'égout, 5 à 10 fois plus cher, n'y aboutit pas ! C'est une situation qu'on rencontre aujourd'hui dans des pays comme la Grèce, où les fonds structurels et de cohésion permettent des investissements rapides et visibles, mais peu coordonnés. En prenant le relais de l'Etat, mais à partir des redevances payées par les usagers, les agences de l'eau ont réalisé la solidarité de bassin par ce qui est le plus important dans une politique de coûts fixes comme les infrastructures, c'est-à-dire l'argent prêté à taux réduit. En faisant une péréquation spatio-temporelle des besoins de financement, elles permettent d'améliorer la performance environnementale de tous les acteurs, sans que ceux qui font des efforts à un moment donné soient seuls face à des emprunts chers, donc à de brutales augmentations de prix des services. Et donc, contrairement à ce que tend à montrer une analyse superficielle de ce qui s'est passé depuis 10 ans environ, les agences ne sont pas responsables d'une augmentation des prix de l'eau, mais au contraire d'une modération de l'augmentation due à des choix politiques nationaux et européens.

Cependant, aujourd'hui, le système des agences doit être réorienté en partie, parce que la résolution des problèmes de pollution ponctuelle en fait surgir d'autres, qui deviennent de plus en plus visibles : pollution diffuse due à l'agriculture, mauvaise gestion des eaux souterraines, surexploitation des ressources en étiage, inondations. La plupart de ces problèmes pourraient être résolus en fait si l'on cessait de protéger les agriculteurs par rapport à la pollution et à la demande en eau dont ils sont responsables, en les faisant rentrer davantage à part entière dans la communauté de politique de l'eau. Reste le cas des inondations, qui doivent être réglées par un autre régime de redevances que celles sur la facture d'eau. Le décret constitutif des agences, ainsi que la jurisprudence du Conseil d'Etat, permettent d'utiliser le thème de la modification du régime des eaux pour créer un système de redevances sur ceux qui aggravent les risques d'inondation. Et une évolution vers la gestion intégrée permettra sans doute d'utiliser les fonds à des actions de maî- 
trise des sols, d'aménagement foncier, plutôt qu'à l'investissement coûteux dans de nouvelles retenues en amont. Mais cela implique aussi une évolution des compétences du personnel des agences. Par ailleurs, il faut bien réfléchir à un système de redevances qui soit effectivement incitatif, et de ce point de vue, le projet "SHON" préparé au milieu des années 1980 par l'agence Seine Normandie était critiquable, car il plaçait le système financier dans la dépendance de l'octroi de permis de construire aggravant les risques [5].

Mais tout ceci ne répond pas à une question qu'on ne manquera pas de se poser : pourquoi faut-il impliquer les agences de l'eau dans les inondations ? Raisonnons à partir de l'un des problèmes majeurs à résoudre, qui est l'économie de la politique d'incitation elle-même : il faut pouvoir récupérer des redevances, mais sans qu'on soit obligé de créer un système de collecte nouveau. Sinon, on risquerait de dépenser plus qu'on ne gagnerait ! Il n'y a alors pratiquement que 2 solutions : la voie de l'impôt local, et celle de la facture d'eau. La première semble a priori s'imposer, dans la mesure où l'imperméabilisation des sols renvoie naturellement à l'impôt foncier. Et d'ailleurs c'est ce qui se passe dans plusieurs pays voisins : en Angleterre, la plupart des ménages payent le service d'assainissement, comme celui de l'eau, par des rates ou des taxes d'habitation ou de collectivités locales. Comme de surcroît les réseaux d'égout sont pratiquement tous unitaires, faire payer le pluvial dans le service de l'assainissement ne pose aucun problème. Et le risque d'inondations rapides ou de plaine est de toutes façons moins important. En Allemagne en revanche, les inondations urbaines dues aux orages sont plus fréquentes, alors que l'assainissement est payé généralement dans les factures d'eau au volume comme en France. Dans ces conditions, plusieurs villes, représentant au moins le quart de la population des Länder de l'ouest, ont créé des taxes sur l'imperméabilisation des sols, pour financer une politique spécifique de pluvial urbain, en respectant les règles de la comptabilité analytique : dès qu'une politique n'est plus marginale financièrement, elle doit avoir sa ligne budgétaire et son mécanisme de financement. Pour les ménages, les taxes sont payées au forfait selon les quartiers, parfois même pas, et seuls les propriétaires fonciers les plus importants, par exemple les entreprises, paient en fonction d'une mesure de l'imperméabilisation. Aux Pays Bas, c'est depuis le Moyen Age que les Wateringues gèrent la maîtrise du drainage et des inondations, par des contributions de type taxes locales. Même l'épuration des eaux usées est payée par des forfaits par famille (excepté pour les célibataires), puisqu'elle est assurée par les Wateringues. Pluvial, protection contre les inondations et assainissement des eaux usées ont toujours été mêlés dans ces institutions, qui sont en plein processus de concentration (il en reste moins de 80 pour 14 millions d'habitants) ; alors que l'eau potable est facturée au volume par d'autres institutions locales.

Il faut cependant prendre en compte un autre critère qui est la taille pertinente du territoire. D'abord, la mise en place d'une gestion spécifique du pluvial urbain, comme les stormwater utilities aux Etats-Unis, fait souvent apparaître assez vite que bien des problèmes sont générés en amont, dans le hinterland rural de la ville. Il faudrait alors en fait élargir au sous-bassin. Ensuite, les municipalités sont souvent responsables d'une bonne part de l'imperméabilisation, par les routes, les parkings, etc., et elles n'aiment pas se taxer ellesmêmes. Mais ne pas le faire compromet l'équité et l'effica- cité de l'incitation économique. Enfin, plus les communes sont petites, et plus les conséquences de choix d'urbanisation qui aggravent les risques risquent d'apparaître dans d'autres communes à l'aval, et donc moins les élus voient pourquoi ils feraient payer des taxes à leurs concitoyens pour régler des problèmes d'autres communes. Ici la France est dans une position extrême, puisque contrairement aux pays précédemment mentionnés, Angleterre, Allemagne, Pays Bas, et même au Portugal, il n'y a pas eu de fusions de communes. Et dans de nombreux problèmes d'environnement, la concurrence entre communes rend la recherche de solutions préventives, de servitudes foncières, encore plus difficile.

\section{VI $\square$ UNE POLITIQUE SUBSIDIAIRE À DEUX NIVEAUX TERRITORIAUX}

C'est pourquoi, en France, on aurait intérêt à développer un système de gestion des inondations passant par les agences de l'eau : on peut très bien ajouter aux factures d'eau des éléments n'ayant pas de rapport avec les volumes consommés, et la taille des agences permet de disposer de budgets d'aide conséquents même si les redevances pluviales initiales sont modestes, et lancer des opérations pilotes. Mais a contrario, la situation politique même des agences est contrainte par les règles "anti-subsidiaires" de notre constitution $[13,19]$ : les agences n'ont ni pouvoir de police, ni maîtrise d'ouvrage, ce qui est excellent pour assurer une médiation bienveillante entre les usagers, et jouer le rôle de l'Etat gardien dont on parlait plus haut, mais qui ne facilite pas la mise en œuvre de la gestion intégrée. C'est là qu'on découvre l'intérêt d'une institution inventée par la loi de 1992, mais complètement oubliée pour l'instant : la communauté locale de l'eau, qui serait au moins une sorte de syndicat intercommunal à contour de sous-bassin versant, chargé de la gestion intégrée de l'eau. Le thème des inondations serait bien évidemment inclus, et d'ailleurs, on peut soupçonner les rédacteurs de la loi de 1992 d'avoir été inspirés par le modèle néerlandais de la Wateringue. A l'autre bout de l'Europe, en Italie, des agences de bassin ont été créées pour certaines rivières inter-régionales (les plus importantes), par la loi de 1989 qui traitait des inondations et des ruissellements érosifs (phénomène gravissime en Italie). D'après mes collègues, elles constituent en ce moment leur légitimité sur la réalisation de cartes de risques, par la coordination des institutions régionales et des entités de ce niveau. Cependant, faute d'un mécanisme de redevances, et faute surtout d'un niveau plus local de saisie des problèmes, cette politique risque de rencontrer des difficultés de mise en œuvre. Une loi plus récente (Galli, 1994) ayant créé, pour une meilleure gestion des services d'eau, l'entité du consorzio idrico (syndicat hydrologique), dont le territoire devrait être le sous-bassin, il reste à voir comment l'articulation des deux niveaux territoriaux permettrait de mieux résoudre les problèmes qui nous préoccupent.

En France, on voit les agences développer, depuis quelques années, toute une panoplie de contrats avec des entités de niveau territorial plus local : rivière, nappe, pays, département, etc. Ce n'est pas un hasard, car il fallait bien résoudre le problème de la coordination des actions, malgré une tendance au saupoudrage des crédits qui résulte de l'impossibilité juridique pour elles d'être maîtres d'ouvrage. Mais il y a plus : c'est que la construction de la confiance des citoyens dans la politique des inondations, ou inversement la lutte 
contre l'anomie sociale, passe par un niveau plus local que les constructions institutionnelles de niveau régional. Tous remarquent ce que le Länderarbeits-gemeinschaft Wasser (LAWA, conférence inter-Länder sur la politique de l'eau) a écrit dans un rapport de synthèse : le plus grave avec les gens qui ont subi une inondation catastrophique, est qu'ils perdent globalement confiance dans la société. Inversement, dans les endroits où les gens sont plus souvent inondés, ils sont préparés, et ils arrivent à vivre avec l'inondation grâce aux solidarités locales qu'ils ont mises au point.

C'est ainsi que dans l'Etat du Parana au Brésil, les services d'urbanisme de l'entité métropolitaine de Curitiba négocient avec les "sans-terre" qui sont venus s'installer dans les zones à risque en bordure de rivières ou dans les marécages en amont de la ville, non pas une exclusion, mais un aménagement des conditions de vie par transferts de droits de construire ${ }^{6}$. Comme dans d'autres Etats brésiliens, les défenseurs de l'idée d'une nouvelle politique de l'eau, basée sur des agences à la française, espèrent qu'elle pourrait démultiplier les procédures participatives et négociées comme celle de Curitiba, qui se développent spontanément. Aux EtatsUnis, nos collègues urbanistes ne plaident pas pour que la Fédération se substitue aux Etats, et encore moins aux collectivités locales, dans la planification des risques, mais pour qu'elle développe des mécanismes économiquement incitatifs à un capacity building local, notamment dans les plans ${ }^{7}$, tout en remettant en cause sa propre politique passée qui tendait à encourager les prises de risques inutiles [1].

Le problème est donc de développer une solidarité préventive, qui passe par des contrats entre divers types d'usagers. En particulier, l'espace agricole peut être organisé pour absorber les crues, à condition qu'une indemnisation correcte des agriculteurs intervienne (polders de la plaine d'Alsace, etc.). Mais il faut aussi que les agriculteurs soient suffisamment organisés entre eux pour pouvoir contracter : le cas du ruissellement érosif en pays de Caux, qui devient au fil des ans de plus en plus régulier et de plus en plus catastrophique, a fait l'objet d'une thèse socio-historique [14] : il en ressort que la sectorisation traditionnelle des administrations, et que l'absence d'intégration entre institutions publiques locales et nationales, ne sont pas seules en cause : l'individualisme paysan, enraciné depuis plusieurs siècles, devient "catastrophique" depuis l'arrivée des techniques modernes de cultures (de drainage des sols) ; l'enchevêtrement des causes des coulées de boues n'est pas perçu, non seulement parce que le territoire impliqué est beaucoup plus vaste que celui dont les habitants ont conscience, mais parce qu'il n'y a pas de structuration communautaire du monde agricole lui permettant de se constituer en interlocuteur des autres victimes des événements climatiques. L'agence de l'eau Seine Normandie reste très marginale dans le jeu des acteurs, puisque les agences n'ont pas officiellement les inondations dans leur champ d'action ; et c'est dommage, car la démarche contractuelle permettrait de décloisonner et de gérer de façon intégrée. Mais elle ne pourrait de toutes façons intervenir qu'en disposant d'un relais sous forme d'une "communauté locale de

6 L'affaire est d'autant plus compliquée que ces zones inondables, les mananciais, constituent les réserves d'eau potable pour l'agglomération, et que la prise en compte des exclus peut seule permettre de limiter les coûts de la ressource future.

7 La réalisation de plans de risques donne notamment aux collectivités locales accès à une réduction à la souscription au système national d'assurances catastrophes, mais la réduction est actuellement plafonnée à ... $2 \%$ : l'eau". C'est ici qu'on peut prendre le sens de cette institution nouvelle imaginée par la loi de 1992. On n'en est pas encore là, mais l'exemple sert à nouveau la thèse selon laquelle, au lieu de camper sur la légitimité constitutionnelle du couple Etat-marché, les administrateurs français devraient encourager le développement d'institutions subsidiaires et communautaires emboîtées pour régler les problèmes relevant du patrimoine commun. Les inondations, c'est de l'eau, c'est du patrimoine commun.

\section{Références}

[1] Burby RAYMOND J., et alii, 1999 : unleashing the power of planning to create disaster-resistant communities, in American Planners Association (APA) Journal, summer.

[2] CiRIACY WANTRUP SiEgFried V. 1985 : Natural ressource economics, selected papers, edited by Richard C. Bishop and Stephen O. Andersen, Westview Press, Boulder.

[3] Hardin GarRetT, 1975 : The tragedy of the commons, in Science, vol. 162.

[4] Saraiva Maria Graça, Correia F. Nunes, Carmo Vitor, 1998 : Avaliação ex-post de medidas não estruturais de defesa contra cheias na bacia hidrográfica da ribeira do Tage, in Quarto congreso da Agua, proceedings of the Lisbon international conference, April.

[5] B. BARRAQUÉ, 1995b : Risques d'inondations : urbanisme réglementaire ou servitude négociée? in SFDE, (infra).

[6] CAPONERA DANTE A. 1992 : Principles of water law and administration. national and international, Balkema, Rotterdam.

[7] Burchi Stefano, 1991 : Current developments and trends in the law and administration of water ressources, in Journal of environmental law. vol. $3 \mathrm{n}^{\circ} 1$, Oxford university press.

[8] Grémion PIERRE, 1976 : Le pouvoir périphérique, bureaucrates et notables dans le système politique français, Le Seuil.

[9] CAUbet Christian, 1995 : Les inondations, le gouvernement, la JICA et les écolos (Brésil), in Ecologie et Politique, n¹5, automne.

[10] TAKEUCHI KUNIYOSHI \& KUNDZEWICZ ZBIGNEw, 1998 : Flood management and damage mitigation, in Water, the looming crisis? Proceedings of the UNESCO International Hydrological Programme conference. June 4-6.

[11] WetZEL VOLKHARD (ed.) 1997 : Management of crisis situations : floods, droughts and institutional aspects, proceedings of the third technical review of EurAqua (European network of fresh water organisations), Rome, Oct. 1996, self-published.

[12] S.F.D.E. (Société française pour le droit de l'environnement), 1995 " Les plans d'exposition aux risques : échec d'une politique publique ?". Revue juridique de l'environnement, III.

[13] B. Barraquê, 1997 : Subsidiarité et politique de l'eau, in Alain Faure (dir.) Territoires et subsidiarité, l'action publique territoriale à la lumière d'un principe controversé, L'Harmattan, Logiques politiques.

[14] CARTIER StÉPHANE, 1999 : Entre recours à l'Etat et recours au marché. principes de solidarité locale face au risque de ruissellement érosif en pays de Caux, thèse de doctorat en sociologie, Université Paris X (LADYSS) - INRA-SAD.

[15] B. BARRAQué (ed) 1995 : Les politiques de l'eau en Europe, La Découverte.

[16] B. BARraqué, 1996 : De l'intérêt général à la politique en réseau, le cas des barrages-réservoirs de la Seine amont, in Annales des Mines, res. ponsabilité et environnement, $\mathrm{n}^{\circ} 2$, série sur les barrages réservoirs

[17] BOURJOL. MAURICE, 1989 : Les biens communaux, Paris, LGDJ, coll Décentralisation et développement local.

[18] Commissariat Général du Plan. Mıchel Matheu (dir), 1997 : Evalua tion du dispositif des agences de l'eau, rapport au gouvernement, Paris. La Documentation Française.

[19] CoRreia F. NunEs (ed), 1998 : Eurowater, institutional dimensions of water management in 5 European countries, Balkema, Rotterdam, 2 vol.

[20] Galland J.P., Dourlens Christine, Theys Jacques, Vidal. Naquet PIERRE, 1991 : Conquête de la sécurité, gestion des risques, actes du séminaire de la DRI du Ministère de l'Equipement, L'Harmattan. 